PROCEEDINGS OF THE

AMERICAN MATHEMATICAL SOCIETY

Volume 130, Number 9, Pages 2669-2677

S 0002-9939(02)06387-6

Article electronically published on March 13, 2002

\title{
OPERATOR HILBERT SPACES WITHOUT THE OPERATOR APPROXIMATION PROPERTY
}

\author{
ALVARO ARIAS
}

(Communicated by N. Tomczak-Jaegermann)

\begin{abstract}
We use a technique of Szankowski to construct operator Hilbert spaces that do not have the operator approximation property, including an example in a noncommutative $L_{p}$ space for $p \neq 2$.
\end{abstract}

\section{INTRODUCTION AND PRELIMINARIES}

A Banach space $X$ has the approximation property, or AP, if the identity operator on $X$ can be approximated uniformly on compact subsets of $X$ by linear operators of finite rank. In the 50's, Grothendieck [G] investigated this property and found several equivalent statements. For example, he proved that $X$ has the AP iff the natural map $J: X^{*} \hat{\otimes} X \rightarrow X^{*} \check{\otimes} X$ is one-to-one ( $\hat{\otimes}$ is the projective tensor product of Banach spaces and $\check{\otimes}$ is the injective tensor product of Banach spaces). However, it remained unknown if every Banach space had the AP until Enflo E constructed the first counterexample in the early 70's. In [S], Szankowski gave a very explicit example of a subspace of $\ell_{p}, 1<p<2$, without the AP. He considered $X=\left(\sum_{n=1}^{\infty} \oplus \ell_{2}^{n}\right)_{p}$, which is isomorphic to $\ell_{p}$, and defined $Z$ to be the closed span of some vectors of length six. He then used a clever combinatorial argument to exploit the difference between the 2-norm of the blocks and the $p$-norm of the sum to prove that $Z$ fails the approximation property. Szankowski's technique is fairly general. In the second section of this paper we will use it to show that the $\ell_{2}$-sum (as defined in [P2]) of row operator spaces has a subspace without the operator space version of the approximation property, or OAP. Since this subspace is a Hilbert space at the Banach space level, it has the Banach approximation property and even a basis. Thus, this is an example of an operator space with the AP but without the OAP. This answers a question of J. Kraus. Furthermore, this is also the first example of an operator space with a basis but without a complete basis. M. Junge suggested that a similar construction using rows in the Schatten $p$-class $S_{p}$ and Rademacher functions in $L_{p}[0,1]$ could lead to an example of a Hilbertian subspace of $L_{p}\left[S_{p}\right]$ failing the OAP. In the third section we verify that this is indeed possible. These are new examples of Hilbertian subspaces of noncommutative $L_{p}$ spaces that are not completely complemented, even if $p>2$.

An operator space $E$ is a Banach space $E$ with an isometric embedding into $B(H)$, the set of all bounded operators on a Hilbert space $H$. Or, equivalently, an

Received by the editors October 10, 2000 and, in revised form, April 12, 2001.

1991 Mathematics Subject Classification. Primary 46B28; Secondary 46B20, 47D15. 
operator space $E$ is a closed subspace of $B(H)$. If $E \subset B\left(H_{1}\right)$ and $F \subset B\left(H_{2}\right)$ are operator spaces, their minimal tensor product $E \otimes_{\min } F$ is the closure of the algebraic tensor product $E \otimes F$ in $B\left(H_{1} \otimes_{2} H_{2}\right)$. A linear map $u: E \rightarrow F$ is completely bounded, or cb, if for every operator space $G$, the map $1_{G} \otimes u: G \otimes_{\min } E \rightarrow G \otimes_{\min } F$ is bounded. The completely bounded norm of $u$, or $\|u\|_{c b}$, is the supremum of $\left\|1_{G} \otimes u\right\|$, where $G$ runs over all operator spaces $G$. It turns out that it is enough to verify that $1_{G} \otimes u$ is bounded when $G$ is $K\left(\ell_{2}\right)$, the set of all compact operators on the Hilbert space $\ell_{2}$, and that $\|u\|_{c b}=\left\|1_{K\left(\ell_{2}\right)} \otimes u\right\|$. The set of all cb-maps from $E$ to $F$ is denoted by $C B(E, F)$. Independently of each other, Blecher and Paulsen [BP] and Effros and Ruan [ER1] gave $E^{*}$, the Banach space dual of $E$, an operator space structure that gives $E^{*} \otimes_{\min } F$ the norm induced by $C B(E, F)$. This indicates that the minimal tensor product is the operator space analogue of the injective tensor product of Banach spaces. In the same papers, Blecher and Paulsen $[\mathrm{BP}$ and Effros and Ruan [ER1 introduced the operator space analogue of the projective tensor product. This is denoted by $E \hat{\otimes} F$ and satisfies $(E \hat{\otimes} F)^{*}=C B\left(E, F^{*}\right)$. We refer to [ER3], [J], and [P3] for more information about operator spaces.

It is well known that the compact subsets of a Banach space $X$ are contained in the convex hull of null sequences in $X$. Since there is a correspondence between null sequences in $X$ and elements of $c_{0} \check{\otimes} X$, it is easy to see that $X$ has the AP iff for every $u \in c_{0} \check{\otimes} X$ and every $\epsilon>0$ there exists a finite rank operator on $X$ such that $\|u-(I \otimes T)(u)\|<\epsilon$. Based on this observation, Effros and Ruan [ER2] said that an operator space $V$ has the operator approximation property, or OAP, if for every $u \in K(H) \otimes_{\min } V$ and every $\epsilon>0$ there exists a finite rank operator $T$ on $V$ such that $\|u-(I \otimes T)(u)\|<\epsilon$. They proved that an operator space $V$ has the OAP if and only if the natural map $J: V^{*} \hat{\otimes} V \rightarrow V^{*} \otimes_{\min } V$ is one-to-one.

The following criterion allows us to check that $J$ is not one-to-one, when $V$ fails the OAP.

Enflo's Criterion. If there exists a sequence of finite rank operators $\beta_{n} \in V^{*} \otimes V$ satisfying:

(i) $\operatorname{trace}\left(\beta_{n}\right)=1$ for every $n \in \mathbb{N}$,

(ii) $\left\|\beta_{n}\right\|_{V^{*} \otimes_{\min } V \rightarrow 0 \text { as } n \rightarrow \infty \text {, and }}$

(iii) $\sum_{n=1}^{\infty}\left\|\beta_{n}-\beta_{n-1}\right\|_{V^{*} \hat{\otimes} V}<\infty$,

then $V$ does not have the OAP.

Indeed, $\beta=\beta_{1}+\sum_{n=2}^{\infty} \beta_{n}-\beta_{n-1}=\lim _{n} \beta_{n}$ belongs to $V^{*} \hat{\otimes} V$ by (iii). $J \beta=0$ by (ii). And since $\operatorname{tr}(\beta)=1, \beta$ is not zero. Hence $J$ is not one-to-one and $V$ fails the OAP.

\section{AN EXAMPLE USING COMPLEX INTERPOLATION}

For each $n \in \mathbb{N}$, let $\Delta_{n}$ be a partition of $\sigma_{n}=\left\{2^{n}, 2^{n}+1,2^{n}+2, \ldots, 2^{n+1}-1\right\}$. Then $\left\{B \in \Delta_{n}: n \in \mathbb{N}\right\}$ is a partition of $\mathbb{N}$. For each $B \in \Delta_{n}$, let $R_{B}$ be the row Hilbert space with orthonormal basis $\left\{e_{j}: j \in B\right\}$. We define $X$ to be the $\ell_{2}$-sum of these row spaces. More precisely, $X$ is the complex interpolation space between $\left(\sum_{n=1}^{\infty} \sum_{B \in \Delta_{n}} \oplus R_{B}\right)_{\infty}$ and $\left(\sum_{n=1}^{\infty} \sum_{B \in \Delta_{n}} \oplus R_{B}\right)_{1}$ of parameter $\theta=\frac{1}{2}$ (see [P2], page 34$)$. That is,

$$
X=\left(\sum_{n=1}^{\infty} \sum_{B \in \Delta_{n}} \oplus R_{B}\right)_{\ell_{2}}=\left(\left(\sum_{n=1}^{\infty} \sum_{B \in \Delta_{n}} \oplus R_{B}\right)_{\infty},\left(\sum_{n=1}^{\infty} \sum_{B \in \Delta_{n}} \oplus R_{B}\right)_{1}\right)_{\frac{1}{2}} .
$$


At the Banach space level, $X$ is a Hilbert space with orthonormal basis $\left\{e_{i}: i \in\right.$ $\mathbb{N}\}$. But at the operator space level, $X$ is a combination of row Hilbert spaces and $O H$, the self-dual operator Hilbert space introduced by Pisier in $[\mathrm{P} 1]$. If $A \subset \mathbb{N}$, let $X_{A}=\overline{\operatorname{span}}\left\{e_{i}: i \in A\right\} \subset X$. It follows from the definition of $X$ that if there exists $n \in \mathbb{N}$ such that $A \subset B$ for some $B \in \Delta_{n}$, then $X_{A}$ is completely isometric to $R_{A}$, the row Hilbert space with orthonormal basis $\left\{e_{i}: i \in A\right\}$. And if for each $n \in \mathbb{N}, A$ has at most one point from each element in $\Delta_{n}$ (i.e., $\operatorname{card}(A \cap B) \leq 1$ for every $B \in \Delta_{n}, n \in \mathbb{N}$ ), then $X_{A}$ is completely isometric to $O H_{A}$.

Let $Z$ be the closed subspace of $X$ spanned by

$$
z_{i}=e_{2 i}-e_{2 i+1}+e_{4 i}+e_{4 i+1}+e_{4 i+2}+e_{4 i+3}, \quad i=1,2, \cdots .
$$

Theorem 1. With the appropriate selection of $\Delta_{n}, Z$ does not have the OAP.

For each $i \in \mathbb{N}$, let $z_{i}^{*}=\frac{1}{2}\left(e_{2 i}^{*}-e_{2 i+1}^{*}\right)$, where the $e_{i}^{*}$ 's are biorthogonal to the $e_{i}$ 's. Then let

$$
\beta_{n}=\frac{1}{2^{n}} \sum_{i \in \sigma_{n}} z_{i}^{*} \otimes z_{i} \quad \text { for } n \geq 2 .
$$

We need to check that the $\beta_{n}$ 's satisfy the conditions of Enflo's criterion.

Condition (i)

This is trivially verified. Since $z_{i}^{*}\left(z_{i}\right)=1$ for every $i \geq 1$, we see that $\operatorname{trace}\left(\beta_{n}\right)=$ $\left(1 / 2^{n}\right) \sum_{i \in \sigma_{n}} z_{i}^{*}\left(z_{i}\right)=\left(1 / 2^{n}\right)\left|\sigma_{n}\right|=1$.

Condition (ii)

Since $\left\|\beta_{n}\right\|_{Z^{*} \otimes_{\min } Z} \leq\left\|\beta_{n}\right\|_{X * \otimes_{\min } X}=\left\|\beta_{n}\right\|_{c b}$, we will estimate the cb-norm of $\beta_{n}: X \rightarrow X$. However, it follows from the definition of $\beta_{n}$ that we only need to estimate the cb-norm of $\beta_{n}: X_{\sigma_{n+1}} \rightarrow X_{\sigma_{n+1}} \cup \sigma_{n+2}$, where $X_{\sigma_{k}}=\operatorname{span}\left\{e_{i}: i \in \sigma_{k}\right\}$. Let $I_{1}: X_{\sigma_{n+1}} \rightarrow R_{\sigma_{n+1}}$ and $I_{2}: R_{\sigma_{n+1} \cup \sigma_{n+2}} \rightarrow X_{\sigma_{n+1} \cup \sigma_{n+2}}$ be the formal identity maps, and let $\tilde{\beta}_{n}: R_{\sigma_{n+1}} \rightarrow R_{\sigma_{n+1} \cup \sigma_{n+2}}$ be $\tilde{\beta}_{n}=\frac{1}{2^{n}} \sum_{i \in \sigma_{n}} z_{i}^{*} \otimes z_{i}$ (that is, $\tilde{\beta}_{n}$ has the same matrix representation as $\beta_{n}$, but it is defined on row operator spaces). Then $\beta_{n}=I_{2} \circ \tilde{\beta}_{n} \circ I_{1}$. Since the $z_{i}$ 's, $i \in \sigma_{n}$, have disjoint support, the $z_{i}^{*}$ 's, $i \in \sigma_{n}$, have also disjoint support, and the row spaces are homogeneous, it is easy to see that $\left\|\tilde{\beta}_{n}\right\|_{c b}=\left\|\tilde{\beta}_{n}\right\|=\frac{1}{2^{n+1}} \sqrt{3}$. We will prove condition (ii) by checking that $\left\|I_{1}\right\|_{c b}\left\|I_{2}\right\|_{c b} \leq \sqrt{2^{n+2}}$.

From the definition of $X$, we see that $X_{\sigma_{n+1}}$ is equal to $\left(\sum_{B \in \Delta_{n+1}} \oplus R_{B}\right)_{\ell_{2}}$, the complex interpolation space $\left(\left(\sum_{B \in \Delta_{n+1}} \oplus R_{B}\right)_{\infty},\left(\sum_{B \in \Delta_{n+1}} \oplus R_{B}\right)_{1}\right)_{\frac{1}{2}}$. It is easy to check that $\left\|I_{1}:\left(\sum_{B \in \Delta_{n+1}} \oplus R_{B}\right)_{\infty} \rightarrow R_{\sigma_{n+1}}\right\|_{c b} \leq \sqrt{\mid \Delta_{n+1}}$ and that $\left\|I_{1}:\left(\sum_{B \in \Delta_{n+1}} \oplus R_{B}\right)_{1} \rightarrow R_{\sigma_{n+1}}\right\|_{c b} \leq 1$. Therefore $\left\|I_{1}\right\|_{c b} \leq\left(\left|\Delta_{n+1}\right|\right)^{\frac{1}{4}}$. Similarly, $\left\|I_{2}\right\|_{c b} \leq\left(\left|\Delta_{n+1}\right|+\left|\Delta_{n+2}\right|\right)^{\frac{1}{4}}$. Since $\left|\Delta_{k}\right| \leq 2^{k}$ for every $k \in \mathbb{N}$, we see that $\left\|I_{1}\right\|_{c b}\left\|I_{2}\right\|_{c b} \leq \sqrt{2^{n+2}}$

Condition (iii)

Using the fact that $z_{i}^{*}=\frac{1}{4}\left(e_{4 i}^{*}+e_{4 i+1}^{*}+e_{4 i+2}^{*}+e_{4 i+3}^{*}\right)$ on $Z$, we get that

$$
\begin{aligned}
& \beta_{n}-\beta_{n-1}=\frac{1}{2^{n+1}} \sum_{i \in \sigma_{n}}\left(e_{2 i}^{*}-e_{2 i+1}^{*}\right) \otimes\left(e_{2 i}-e_{2 i+1}+e_{4 i}+e_{4 i+1}+e_{4 i+2}+e_{4 i+3}\right) \\
& -\frac{1}{2^{n+1}} \sum_{i \in \sigma_{n-1}}\left(e_{4 i}^{*}+e_{4 i+1}^{*}+e_{4 i+2}^{*}+e_{4 i+3}^{*}\right) \otimes\left(e_{2 i}-e_{2 i+1}+e_{4 i}+e_{4 i+1}+e_{4 i+2}+e_{4 i+3}\right)
\end{aligned}
$$




$$
=\frac{1}{2^{n+1}} \sum_{i \in \sigma_{n-1}}\left\{\begin{array}{l}
e_{4 i}^{*} \otimes\left(e_{4 i}-e_{4 i+1}+e_{8 i}+e_{8 i+1}+e_{8 i+2}+e_{8 i+3}-e_{2 i}+e_{2 i+1}-e_{4 i}-e_{4 i+1}-e_{4 i+2}-e_{4 i+3}\right) \\
e_{4 i+1}^{*} \otimes\left(-e_{4 i}+e_{4 i+1}-e_{8 i}-e_{8 i+1}-e_{8 i+2}-e_{8 i+3}-e_{2 i}+e_{2 i+1}-e_{4 i}-e_{4 i+1}-e_{4 i+2}-e_{4 i+3}\right) \\
e_{4 i+2}^{*} \otimes\left(e_{4 i+2}-e_{4 i+3}+e_{8 i+4}+e_{8 i+5}+e_{8 i+6}+e_{8 i+7}-e_{2 i}+e_{2 i+1}-e_{4 i}-e_{4 i+1}-e_{4 i+2}-e_{4 i+3}\right) \\
e_{4 i+3}^{*} \otimes\left(-e_{4 i+2}+e_{4 i+3}-e_{8 i+4}-e_{8 i+5}-e_{8 i+6}-e_{8 i+7}-e_{2 i}+e_{2 i+1}-e_{4 i}-e_{4 i+1}-e_{4 i+2}-e_{4 i+3}\right) .
\end{array}\right.
$$

Note that after cancellation, each of the vectors in the brace has nine terms. Two of them cancel out and two are equal. Then we can write each of them as a linear combination of nine basis vectors. Eight of them have coefficients equal to \pm 1 and the other has a coefficient equal to \pm 2 .

Szankowski defined nine functions $f_{k}: \mathbb{N} \rightarrow \mathbb{N}, k \leq 9$, to index these vectors. Let $n=4 i+l$ and $l=0,1,2,3$. Then $f_{1}(4 i+l)=2 i$ and $f_{2}(4 i+l)=2 i+1$. For $k=3,4,5, f_{k}(4 i+l)=4 i+[(l+1) \bmod 4]$. For $l=0,1, f_{6}(4 i+l)=8 i$, $f_{7}(4 i+l)=8 i+1, f_{8}(4 i+l)=8 i+2$, and $f_{9}(4 k+l)=8 i+3$. And finally, for $l=2,3, f_{6}(4 i+l)=8 i+4, f_{7}(4 i+l)=8 i+5, f_{8}(4 i+l)=8 i+6$, and $f_{9}(4 k+l)=8 i+7$. Then we have

$$
\beta_{n}-\beta_{n-1}=\frac{1}{2^{n+1}} \sum_{j \in \sigma_{n+1}} e_{j}^{*} \otimes y_{j}
$$

where $y_{j}=\sum_{k=1}^{9}=\lambda_{j, k} e_{f_{k}(j)} \in Z$. Recall that eight of the $\lambda_{j, k}$ 's have absolute value equal to one, and one has absolute value equal to 2 .

The following lemma of Szankowski provides the key combinatorial argument (see $[\mathrm{S}$ ] and $[\mathrm{LT}]$, page 108).

Lemma 2 (Szankowski). There exist partitions $\Delta_{n}$ and $\nabla_{n}$ of $\sigma_{n}$ into disjoint sets, and a sequence $m_{n} \geq 2^{\frac{n}{8}-2}, n=2,3, \ldots$, so that

$1 \forall A \in \nabla_{n}, m_{n} \leq \operatorname{card}(A) \leq 2 m_{n}$,

$2 \forall A \in \nabla_{n}, \forall B \in \Delta_{n}, \operatorname{card}(A \cap B) \leq 1$,

$3 \forall A \in \nabla_{n}, \forall 1 \leq k \leq 9, f_{k}(A)$ is contained in an element of $\Delta_{n-1}, \Delta_{n}$, or $\Delta_{n+1}$.

(Notice that $f_{k}\left(\sigma_{n}\right) \subset \sigma_{n-1}$ for $k=1,2, f_{k}\left(\sigma_{n}\right) \subset \sigma_{n}$ for $k=3,4,5$, and $f_{k}\left(\sigma_{n}\right) \subset \sigma_{n+1}$ for $k=6,7,8,9$.)

Since $\nabla_{n+1}$ is a partition of $\sigma_{n+1}$, we have that

$$
\beta_{n}-\beta_{n-1}=\frac{1}{2^{n+1}} \sum_{A \in \nabla_{n+1}}\left[\sum_{j \in A} e_{j}^{*} \otimes y_{j}\right]
$$

Lemma 3. For every $A \in \nabla_{n+1},\left\|\sum_{j \in A} e_{j}^{*} \otimes y_{j}\right\|_{Z^{*} \hat{\otimes} Z} \leq 18(\operatorname{card}(A))^{\frac{3}{4}}$.

The last condition of Enflo's criterion follows immediatedly from (1), Lemma 2, and Lemma 3. Indeed,

$$
\begin{aligned}
\left\|\beta_{n}-\beta_{n-1}\right\|_{Z * \hat{\otimes} Z} & \leq \frac{1}{2^{n+1}} \operatorname{card}\left(\nabla_{n+1}\right) 18 \max _{A \in \nabla_{n+1}} \operatorname{card}(A)^{\frac{3}{4}} \\
& \leq \frac{1}{2^{n+1}} \frac{2^{n+1}}{m_{n+1}} 18\left(2 m_{n+1}\right)^{\frac{3}{4}} \leq \frac{36}{m_{n+1}^{\frac{1}{4}}},
\end{aligned}
$$

which is clearly summable.

We only need to prove Lemma 3. For this, we need the result of Pisier (see remark 2.11 of $[\overline{\mathrm{P} 1}])$ that $C B\left(R_{n}, O H_{n}\right)=S_{4}^{n}$, where $S_{4}^{n}$ is the Schatten 4-class. 
Consequently, if $S: O H_{n} \rightarrow R_{n}$, then $\|S\|_{O H_{n} \hat{\otimes} R_{n}}=\|S\|_{S_{4 / 3}^{n}}$. In particular, if $I: O H_{n} \rightarrow R_{n}$ is the formal identity, $\|I\|_{O H_{n} \hat{\otimes} R_{n}}=n^{3 / 4}$.

Proof of Lemma 3. The element $\gamma=\sum_{j \in A} e_{j}^{*} \otimes y_{j} \in X^{*} \hat{\otimes} Z$ induces a finite rank map $\gamma: X \rightarrow Z$. The restriction of $\gamma$ to $Z$ is the map $\alpha=\gamma_{\mid Z}: Z \rightarrow Z$, which clearly satisfies $\alpha=\sum_{j \in A} q\left(e_{j}^{*}\right) \otimes y_{j} \in Z^{*} \hat{\otimes} Z$, where $q=\left(\iota_{Z}\right)^{*}: X^{*} \rightarrow Z^{*}$ is the adjoint of the inclusion $\iota_{Z}: Z \rightarrow X$. Since $\left(Z^{*} \hat{\otimes} Z\right)^{*}=C B\left(Z^{*}, Z^{*}\right)$, we have that $\|\alpha\|_{Z^{*} \hat{\otimes} Z}=\sup \left\{|\langle T, \alpha\rangle|: T: Z^{*} \rightarrow Z^{*}, \quad\|T\|_{c b} \leq 1\right\}$, where $\langle\cdot, \cdot\rangle$ is the trace duality.

We will see that we can factor $\alpha$ through the formal identity map $I: O H_{A} \rightarrow R_{A}$, where $R_{A}$ is the row Hilbert space with basis $\left\{\delta_{j}: j \in A\right\}$. Recall that the projective tensor norm of $I: O H_{A} \rightarrow R_{A}$, viewed as an element of $O H_{A} \hat{\otimes} R_{A}$, is equal to $(\operatorname{card}(A))^{\frac{3}{4}}$.

Let $\Psi: R_{A} \rightarrow Z$ be the map defined by $\Psi\left(\delta_{j}\right)=y_{j}$. We claim that $\|\Psi\|_{c b} \leq 18$. Indeed, if $a_{j} \in B(\mathcal{H})$ for $j \in A$, then

$$
\sum_{j \in A} a_{i} \otimes y_{j}=\sum_{j \in A} a_{j} \otimes \sum_{k=1}^{9} \lambda_{j, k} e_{f_{k}(j)}=\sum_{k=1}^{9}\left[\sum_{j \in A} \lambda_{j, k} a_{j} \otimes e_{f_{k}(j)}\right] .
$$

It follows from (3) of Lemma 2 that $\left\{f_{k}(j): j \in A\right\} \subset B$ for some $B$ in $\Delta_{n}, \Delta_{n+1}$, or $\Delta_{n+2}$. Then the definition of $X$ implies that the span of the $e_{f_{k}(j)}$ 's for $j \in A$ is a row operator space. Hence,

$$
\left\|\sum_{j \in A} a_{i} \otimes y_{j}\right\| \leq(9)(2)\left\|\sum_{j \in A} a_{j} a_{j}^{*}\right\|^{\frac{1}{2}}=18\left\|\sum_{j \in A} a_{j} \otimes \delta_{j}\right\| .
$$

Let $X_{A}=\operatorname{span}\left\{e_{j}: j \in A\right\}$. By (2) of Lemma 2, all the elements of $A$ belong to different elements of the partition $\Delta_{n+1}$. This implies that $X_{A}$ is completely isometric to $O H_{A}$. Let $P_{A}: X \rightarrow X_{A}$ be the completely contractive projection onto $X_{A}$, and let $I: X_{A} \rightarrow R_{A}$ be the formal identity. Then we have that

$$
\alpha=\Psi \circ I \circ P_{A} \circ \iota_{Z} .
$$

If $T: Z^{*} \rightarrow Z^{*}$ is completely bounded, then

$$
\begin{aligned}
|\langle T, \alpha\rangle| & =\left|\operatorname{tr}\left(T^{*} \circ \alpha\right)\right|=\left|\operatorname{tr}\left(T^{*} \circ \Psi \circ I \circ P_{A} \circ \iota_{Z}\right)\right|=\left|\operatorname{tr}\left(P_{A} \circ \iota_{Z} \circ T^{*} \circ \Psi \circ I\right)\right| \\
& \leq\left\|P_{A} \circ \iota_{Z} \circ T^{*} \circ \Psi\right\|_{c b}\|I\|_{O H_{A} \hat{\otimes} R_{A}} \leq 18\|T\|_{c b}(\operatorname{card}(A))^{\frac{3}{4}} .
\end{aligned}
$$

This finishes the proof of Lemma 3 .

Remarks. We can replace the $\ell_{2}$ sum of $R_{n}$ 's by the $\ell_{2}$ sum of $C_{n}$ 's or by the $\ell_{2}$ sum of any other family of homogeneous Hilbert spaces which is "far" from $O H$ (e.g., $R_{p}^{n}=\left(R_{n}, C_{n}\right)_{\frac{1}{p}}, p \neq 2$ ). The same proof gives that all of these spaces have subspaces failing the operator approximation property. We can also replace the $\ell_{2}$ sum of $R_{n}$ 's by the $\ell_{p}$ sum of $R_{n}$ 's. However, when $p \neq 2$, then $X$ is no longer a Hilbert space. The subspace $Z$ of $X$ will fail the operator approximation property, but Szankowski's theorem actually gives that $Z$ already fails the Banach approximation property. 


\section{An example in noncommutative $L_{p}$}

We recall standard facts about noncommutative $L_{p}$ spaces. $S_{p}$ is the Schatten $p$ class on $\ell_{2}$ with the operator space structure induced by the complex interpolation $S_{p}=\left(S_{\infty}, S_{1}\right)_{\frac{1}{p}}$ (see [P2]). $R_{p}=\overline{\operatorname{span}}\left\{e_{1 n}: n \in \mathbb{N}\right\}$ is the row of $S_{p}$, and $C_{p}=$ $\overline{\operatorname{span}}\left\{e_{n 1}: n \in \mathbb{N}\right\}$ is the column of $S_{p}$. Both of them are taken with the operator space structure they inherit from $S_{p}$. It follows from Lemma 1.7 of [P2] that the operator space structure of any operator space $E$ is determined by $S_{p}[E]$, the noncommutative $E$-valued $S_{p}$-space. In particular, the operator space structures of $R_{p}$ and $S_{p}$ are determined by $S_{p}\left[R_{p}\right]$ and $S_{p}\left[C_{p}\right]$. That is, if $a_{n} \in S_{p}$ is a finite family, then

$$
\left\|\sum_{n} a_{n} \otimes e_{1 n}\right\|_{S_{p}\left[R_{p}\right]}=\left\|\left(\sum_{n} a_{n} a_{n}^{*}\right)^{\frac{1}{2}}\right\|_{S_{p}}
$$

and

$$
\left\|\sum_{n} a_{n} \otimes e_{n 1}\right\|_{S_{p}\left[C_{p}\right]}=\left\|\left(\sum_{n} a_{n}^{*} a_{n}\right)^{\frac{1}{2}}\right\|_{S_{p}} .
$$

We will also consider $R_{p}^{n}$ and $C_{p}^{n}$, the row and column of $S_{p}^{n}$; and more generally, if $B$ is a subset of $\mathbb{N}, R_{p}^{B}$ and $C_{p}^{B}$ are the row and column of $S_{p}^{B}$, the Schatten $p$-class on the Hilbert space with orthonormal basis indexed by $B$.

The operator space structure of $L_{p}[0,1]$ is determined by $S_{p}\left[L_{p}\right]$. We note that by Proposition 2.1 of [P2], $S_{p}\left[L_{p}\right]$ is completely isometric to the more familiar $L_{p}\left[S_{p}\right]$. We will consider $\mathcal{R}_{p} \subset L_{p}[0,1]$, the subspace generated by the Rademacher functions $\left(\epsilon_{n}\right)_{n \in \mathbb{N}}$, with the operator space structure inherited from $L_{p}$. It is known that the operator space structure of $\mathcal{R}_{p}$ is determined by the noncommutative Khintchine's inequalities of [L-P] and [L-PP]. If $p \geq 2, \mathcal{R}_{p}$ is completely isomorphic to $R_{p} \cap C_{p}$ and $\mathcal{R}_{p}^{n}$ is completely isomorphic to $R_{p}^{n} \cap C_{p}^{n}$, with a constant depending only on $p$. If $1 \leq p \leq 2, \mathcal{R}_{p}$ is completely isomorphic to $R_{p}+C_{p}$ and $\mathcal{R}_{p}^{n}$ is completely isomorphic to $R_{p}^{n}+C_{p}^{n}$, with a constant depending only on $p$ (see [P2], Section 8.4, for details).

Recall that the classical Khintchine's inequality states that there exist constants $A_{p}$ and $B_{p}$ such that for any square summable sequence $\left(\alpha_{n}\right)$

$$
A_{p}\left\|\sum_{n} \alpha_{n} \epsilon_{n}\right\|_{2} \leq\left\|\sum_{n} \alpha_{n} \epsilon_{n}\right\|_{p} \leq B_{p}\left\|\sum_{n} \alpha_{n} \epsilon_{n}\right\|_{2} .
$$

We will now construct a Hilbertian subspace of $L_{p}\left[S_{p}\right]$ as a combination of Rademacher functions in $L_{p}$ and the row of $S_{p}$. The idea is to put a finite set of Rademacher functions in the $(1,1)$ row of $S_{p}$, then to put another finite set of Rademacher functions in the $(1,2)$ row of $S_{p}$, a third finite set of Rademacher functions in the $(1,3)$ row of $S_{p}$, and so on. At the end we get a space $X$ which plays the

same role as the space $X$ of the previous section. To make the construction more precise, recall that for each $n \in \mathbb{N}, \Delta_{n}$ is a partition of $\sigma_{n}=\left\{2^{n}, 2^{n}+1, \ldots, 2^{n+1}\right\}$. Then $\mathcal{P}=\left\{B \in \Delta_{n}: n \in \mathbb{N}\right\}$ is a partition of $\mathbb{N}$. For convenience, we index the row of $S_{p}$ by the countable set $\mathcal{P}$. That is, $R_{p}=\overline{\operatorname{span}}\left\{e_{1 B}: B \in \mathcal{P}\right\}$. Define

$$
X=\overline{\operatorname{span}}\left\{e_{1 B} \otimes \epsilon_{k}: B \in \mathcal{P}, k \in B\right\} \subset S_{p}\left[L_{p}\right] \equiv L_{p}\left[S_{p}\right] .
$$


Alternatively, define $e_{k}:=e_{1 B} \otimes \epsilon_{k} \in S_{p}\left[L_{p}\right]$, where $B \in \mathcal{P}$ is the element in the partition that contains $k \in \mathbb{N}$, and let $X$ be the closed span of the $e_{k}$ 's.

Proposition 4. At the Banach space level, $X$ is isomorphic to a Hilbert space.

Proof. An element $f \in X$ has the form $f=\sum_{B \in \mathcal{P}} e_{1 B} \otimes f_{B}$, where $f_{B}$ belongs to $\operatorname{span}\left\{e_{k}: k \in B\right\}=\mathcal{R}_{p}^{B}$. Since $S_{p}\left[L_{p}\right]$ is completely isometric to $L_{p}\left[S_{p}\right]$, we view $f:[0,1] \rightarrow S_{p}$ as an $S_{p}$-valued function with norm

$$
\|f\|_{L_{p}\left[S_{p}\right]}=\left(\int_{0}^{1}\|f(t)\|_{S_{p}}^{p} d t\right)^{\frac{1}{p}}=\left[\int_{0}^{1}\left(\sum_{B \in \mathcal{P}}\left|f_{B}(t)\right|^{2}\right)^{\frac{p}{2}} d t\right]^{\frac{1}{p}} .
$$

If $p \geq 2$, then $\|f\|_{L_{p}\left[S_{p}\right]} \geq\left(\int_{0}^{1}\left(\sum_{B \in \mathcal{B}}\left|f_{B}(t)\right|^{2}\right)^{\frac{2}{2}} d t\right)^{\frac{1}{2}}=\sqrt{\sum_{B \in \mathcal{P}}\left\|f_{B}\right\|_{2}^{2}}$. To check the opposite direction, we use that $L_{p}\left[S_{p}\right]$ has type 2 , and that $\sum_{B \in \mathcal{P}} e_{1 B} \otimes f_{B}$ is an unconditional sum. Then, $\|f\|_{S_{p}\left[L_{p}\right]} \leq C \sqrt{\sum_{B \in \mathcal{P}}\left\|f_{B}\right\|_{p}^{2}}$. Since $f_{B} \in \mathcal{R}_{p}^{B}$, it follows from (2) that $\left\|f_{B}\right\|_{p} \leq B_{p}\left\|f_{B}\right\|_{2}$. Therefore, $\|f\|_{S_{p}\left[L_{p}\right]}$ is equivalent to $\sqrt{\sum_{B \in \mathcal{P}}\left\|f_{B}\right\|_{2}^{2}}$, which is the norm of a Hilbert space.

The case $1 \leq p \leq 2$ is similar. We first check that $\|f\|_{L_{p}\left[S_{p}\right]} \leq \sqrt{\sum_{B \in \mathcal{P}}\left\|f_{B}\right\|_{2}^{2}}$. Then we use the cotype 2 of $L_{p}\left[S_{p}\right]$ and the Rademacher estimate of (2) to conclude that $\|f\|_{L_{p}\left[S_{p}\right]} \geq C \sqrt{\sum_{B \in \mathcal{P}}\left\|f_{B}\right\|_{2}^{2}}$.

The operator space structure of $X$ can be described easily. The blocks of $X$ are indexed by $B \in \mathcal{P}$, and they are completely isometric to $\operatorname{span}\left\{\epsilon_{k}: k \in B\right\}=\mathcal{R}_{p}^{B}$. When $p \geq 2, \mathcal{R}_{p}^{B}$ is completely isomorphic (with constant depending only on $p$ ) to $R_{p}^{B} \cap C_{p}^{B}$, and when $1 \leq p \leq 2, \mathcal{R}_{p}^{B}$ is completely isomorphic to $R_{p}^{B}+C_{p}^{B}$. Since the sum is taken in an $R_{p}$ sense, we obtain the following complete isomorphisms:

$$
\begin{array}{ll}
X \approx\left(\sum_{n=1}^{\infty} \sum_{B \in \Delta_{n}} \oplus\left[R_{p}^{B} \cap C_{p}^{B}\right]\right)_{R_{p}} \quad \text { for } p \geq 2, \\
X \approx\left(\sum_{n=1}^{\infty} \sum_{B \in \Delta_{n}} \oplus\left[R_{p}^{B}+C_{p}^{B}\right]\right)_{R_{p}} & \text { for } 1 \leq p \leq 2 .
\end{array}
$$

To apply Enflo's criterion, we need the following estimates.

Lemma 5. Let $1 \leq p \leq \infty$. Then the normed spaces $C B\left(C_{p}^{n}, R_{p}^{n}\right), C B\left(R_{p}^{n}, C_{p}^{n}\right)$, $C B\left(C_{p}^{n}, R_{p}^{n} \cap C_{p}^{n}\right)$, and $C B\left(R_{p}^{n}, R_{p}^{n} \cap C_{p}^{n}\right)$ are isomorphic to $S_{r}^{n}$, the Schatten $r$-class, where $r$ satisfies $\frac{1}{r}=\left|\frac{1}{p}-\frac{1}{2}\right|$.

Proof. It is enough to prove that $C B\left(C_{p}^{n}, R_{p}^{n}\right)$ is isomorphic to $S_{r}^{n}$. The proof for the second space is similar, and the proof for the last two spaces follows from the first two. By duality, it is enough to assume that $1 \leq p \leq 2$. Let $T \in C B\left(C_{p}^{n}, R_{p}^{n}\right)$. Write $T=U D V$, where $U: R_{p}^{n} \rightarrow R_{p}^{n}$ and $V: C_{p}^{n} \rightarrow C_{p}^{n}$ are unitary, and $D: C_{p}^{n} \rightarrow R_{p}^{n}$ is the diagonal operator $D e_{i 1}=\lambda_{i} e_{1 i}$ with $\lambda_{i}$ equal to the $i$ th singular number of $T$. Since $R_{p}^{n}$ and $C_{p}^{n}$ are homogeneous Hilbert spaces, we conclude that $\|T\|_{c b}=\|D\|_{c b}$. By Lemma 1.7 of [P2],

$$
\|D\|_{c b}=\sup \left\{\frac{\left\|\sum_{i \leq n} \lambda_{i} e_{1 i} \otimes a_{i}\right\|_{R_{p}^{n}\left[S_{p}\right]}}{\left\|\sum_{i \leq n} e_{i 1} \otimes a_{i}\right\|_{C_{p}^{n}\left[S_{p}\right]}}: a_{i} \in S_{p}, \quad i \leq n\right\} \text {. }
$$


It is well known that, for $1 \leq p \leq 2,\left\|\sum_{i \leq n} \lambda_{i} e_{1 i} \otimes a_{i}\right\| \leq\left(\sum_{i \leq n}\left|\lambda_{i}\right|^{p}\left\|a_{i}\right\|_{S_{p}}^{p}\right)^{\frac{1}{p}}$ and $\left\|\sum_{i \leq n} e_{i 1} \otimes a_{i}\right\| \geq\left(\sum_{i \leq n}\left\|a_{i}\right\|_{S_{p}}^{2}\right)^{\frac{1}{2}}$. Moreover, if $a_{i}=b_{i} e_{i 1} \in S_{p}^{n}$ for some scalars $b_{i} \in \mathbb{C}$, the inequalities are attained. Since $\left\|a_{i}\right\|_{S_{p}}=\left|b_{i}\right|$, we have

$$
\|D\|_{c b}=\sup \left\{\left(\sum_{i \leq n}\left|\lambda_{i} b_{i}\right|^{p}\right)^{\frac{1}{p}}: \sqrt{\sum_{i \leq n}\left|b_{i}\right|^{2}} \leq 1\right\}=\left(\sum_{i \leq n}\left|\lambda_{i}\right|^{r}\right)^{\frac{1}{r}}=\|T\|_{S_{r}^{n}} .
$$

This finishes the proof.

Since $C B\left(C_{p}^{n}, R_{p}^{n} \cap C_{p}^{n}\right)$ is the dual of $\left(R_{p}^{n}\right)^{*} \hat{\otimes}\left(R_{p}^{n}+C_{p}^{n}\right)$, it follows from Lemma 5 that for every $T \in\left(R_{p}^{n}\right)^{*} \hat{\otimes}\left(R_{p}^{n}+C_{p}^{n}\right)$ we have $\|T\|_{\left(R_{p}^{n}\right) * \hat{\otimes}\left(R_{p}^{n}+C_{p}^{n}\right)}=\|T\|_{S_{r^{\prime}}^{n}}$, where $\frac{1}{r}+\frac{1}{r^{\prime}}=1$. In particular, if $T=I_{n}$ is the "formal" identity map, then

$$
\left\|I_{n}\right\|_{\left(R_{p}^{n}\right) * \hat{\otimes}\left(R_{p}^{n}+C_{p}^{n}\right)}=n^{\frac{1}{r^{\prime}}} .
$$

Similarly,

$$
\left\|I_{n}\right\|_{\left(R_{p}^{n} \cap C_{p}^{n}\right)^{*} \hat{\otimes} R_{p}^{n}}=n^{\frac{1}{r^{\prime}}}
$$

With these estimates, we can follow Szankowski's construction to obtain the following theorem. We sketch the proof.

Theorem 6. For every $1 \leq p<\infty, p \neq 2$, there exists a Hilbertian subspace of $L_{p}\left[S_{p}\right]$ without the operator approximation property.

Recall that $X$ is the closed span of $e_{k}:=e_{1 B} \otimes \epsilon_{k}, k \in \mathbb{N}$. For each $i=1,2, \ldots$, let $z_{i}=e_{2 i}-e_{2 i+1}+e_{4 i}+e_{4 i+1}+e_{4 i+2}+e_{4 i+3}$ and $z_{i}^{*}=\frac{1}{2}\left(e_{2 i}^{*}-e_{2 i+1}^{*}\right)$, where the $e_{i}^{*}$ 's are biorthogonal to the $e_{i}$ 's. For each $n \geq 2$, let $\beta_{n}=\frac{1}{2^{n}} \sum_{i \in \sigma_{n}} z_{i}^{*} \otimes z_{i}$. Finally, let $Z$ be the closed span of the $z_{i}$ 's. The claim is that there exist partitions $\left(\Delta_{n}\right)_{n}$ such that $Z$ fails the OAP. We prove this by checking the three conditions of Enflo's criterion. The first one is trivial. The second one follows easily. We estimate the cb-norm of $\beta_{n}$ through the cb-norm of $\tilde{\beta}_{n}$, which has the same matrix representation as $\beta_{n}$ but is defined on $R_{p}$, the row of $S_{p}$.

We use Szankowski's partitions of Lemma 2 to check the third condition of Enflo's criterion for $1 \leq p<2$. Recall that $\beta_{n}-\beta_{n-1}=\frac{1}{2^{n+1}} \sum_{j \in \sigma_{n+1}} e_{j}^{*} \otimes y_{j}=$ $\frac{1}{2^{n+1}} \sum_{A \in \nabla_{n+1}}\left[\sum_{j \in A} e_{j}^{*} \otimes y_{j}\right]$, where $y_{j}=\sum_{k=1}^{9} \lambda_{j, k} e_{f_{k}(j)} \in Z$ for some $\left|\lambda_{j, k}\right|=1$ or 2. Let $A \in \nabla_{n+1}$. It follows from Lemma 2 (2) that $X_{A}=\operatorname{span}\left\{e_{i}: i \in A\right\}$ is completely isomorphic to $R_{p}^{A}$. And if $\Psi: R_{p}^{A}+C_{p}^{A} \rightarrow Z$ is defined by $\Psi\left(\delta_{j}\right)=y_{j}$, it follows from Lemma $2(3)$ that $\|\Psi\|_{c b} \leq 18$. Note that

$$
\sum_{j \in A} e_{j}^{*} \otimes y_{j}=\sum_{j \in A} e_{j}^{*} \otimes \Psi\left(\delta_{j}\right)=(I \otimes \Psi) \sum_{j \in A} e_{j}^{*} \otimes \delta_{j},
$$

and that $\sum_{j \in A} e_{j}^{*} \otimes \delta_{j}$ is the "formal" identity map on $\left(R_{p}^{A}\right)^{*} \hat{\otimes}\left(R_{p}^{A}+C_{p}^{A}\right)$. Then it follows from (3) that

$$
\left\|\sum_{j \in A} e_{j}^{*} \otimes y_{j}\right\|_{Z^{*} \hat{\otimes} Z} \leq\|\Psi\|_{c b}\left\|\sum_{j \in A} e_{j}^{*} \otimes \delta_{j}\right\|_{\left(R_{p}^{A}\right) * \hat{\otimes}\left(R_{p}^{A}+C_{p}^{A}\right)} \leq 18(\operatorname{card}(A))^{\frac{1}{r^{\prime}}},
$$

where $\frac{1}{r}+\frac{1}{r^{\prime}}=1$ and $\frac{1}{r}=\left|\frac{1}{p}-\frac{1}{2}\right|$. Now, we easily check $\sum_{n}\left\|\beta_{n}-\beta_{n-1}\right\|_{Z^{*} \hat{\otimes} Z}<\infty$.

To prove the third condition of Enflo's criterion for $p>2$, we consider the variation of Lemma 2 described in [LT], page 111. Namely, we find partitions $\Delta_{n}$, $\nabla_{n}$ such that every $A \in \nabla_{n}$ is contained in some element of $\Delta_{n}$ while, for every 
$A \in \nabla_{n}, k=1, \ldots, 9$, and $B$ in $\Delta_{n-1}, \Delta_{n}$, or $\Delta_{n+1}, \operatorname{card}\left(B \cap f_{k}(A)\right) \leq 1$. Then, if $A \in \nabla_{n+1}, X_{A}$ is completely isomorphic to $R_{p}^{A} \cap C_{p}^{A}$, and if $\Psi: R_{p}^{A} \rightarrow Z$ is defined by $\Psi\left(\delta_{j}\right)=y_{j}$, then $\|\Psi\|_{c b} \leq 18$. Since $\sum_{j \in A} e_{j}^{*} \otimes \delta_{j}$ is the "formal" identity map

on $\left(R_{p}^{A} \cap C_{p}^{A}\right)^{*} \hat{\otimes} R_{p}^{A}$, it follows from (4) that $\left\|\sum_{j \in A} e_{j}^{*} \otimes y_{j}\right\|_{Z^{*} \hat{\otimes} Z} \leq 18(\operatorname{card}(A))^{\frac{1}{r^{\prime}}}$, and the third condition of Enflo's criterion follows.

\section{ACKNOWLEDGMENT}

The author thanks Marius Junge for suggesting the use of Rademacher functions and rows in $S_{p}$ to find a Hilbertian subspace of noncommutative $L_{p}$ spaces failing the OAP.

\section{REFERENCES}

[BP] D. Blecher and V. Paulsen, Tensor products of operator spaces, Journal of Functional Analysis 99 (1991), 262-292. MR 93d:46095

[ER1] E. Effros and Z.J. Ruan, A new approach to operator spaces, Canadian Math. Bull 34 (1991), 329-337. MR 93a:47045

[ER2] E. Effros and Z.J. Ruan, On approximation properties for operator spaces, International Journ. Math 1 (1990), 163-187. MR 92g:46089

[ER3] E. Effros and Z.J. Ruan, Operator spaces, Oxford University Press, 2000. CMP 2001:05

[E] P. Enflo, A counterexample to the approximation property in Banach spaces, Acta Math. 130 (1973), 309-317. MR 53:6288

[J] M. Junge, Factorization theory for operator spaces, Habilitation thesis, Kiel University, 1996.

[G] A. Grothendieck, Produits tensoriels topoligiques et espaces nucleaires, Mem. Amer. Math. Soc. 16 (1955). MR 17:763c

[LT] J. Lindenstrauss and L. Tzafriri, Classical Banach spaces II, function spaces, SpringerVerlag, Berlin, 1979. MR 81c:46001

[L-P] F. Lust-Piquard, Inégalités de Khintchine dans $C_{p}(1<p<\infty)$, C. R. Acad. Sci. Paris Sr. I Math. 303 (1986), 289-292. MR 87i:47032

[L-PP] F. Lust-Piquard and G. Pisier, Noncommutative Khintchine and Paley inequalities, Ark. Mat. 29 (1991), 241-260. MR 94b:46011

[P1] G. Pisier, The operator Hilbert space $\mathrm{OH}$, complex interpolation and tensor norms, Mem. Amer. Math. Soc. 585122 (1996), 1-103. MR 97a:46024

[P2] G. Pisier, Non-commutative vector valued $L_{P}$-spaces and completely -summing maps., Soc. Math. France, Asterisque 247 (1998), 1-131. MR 2000a:46108

[P3] G. Pisier, An introduction to the theory of operator spaces (to appear).

[S] A. Szankowski, Subspaces without approximation property, Israel Journal of Math. 30 (1978), 123-129. MR 80b:46032

Division of Mathematics and Statistics, The University of Texas at San Antonio, San Antonio, Texas 78249

E-mail address: arias@math.utsa.edu 